\title{
Robustness Analysis of Hybrid Stochastic Neural Networks with Neutral Terms and Time-Varying Delays
}

\author{
Chunmei Wu, ${ }^{1,2}$ Junhao $\mathrm{Hu}^{1}$ and ${\operatorname{Yan~} \mathrm{Li}^{3}}^{3}$ \\ ${ }^{1}$ College of Mathematics and Statistics, South Central University for Nationalities, Wuhan 430074, China \\ ${ }^{2}$ Department of Mathematics and Computer Science, Liuzhou Teachers College, Liuzhou 546100, China \\ ${ }^{3}$ College of Science, Huazhong Agriculture University, Wuhan 430070, China
}

Correspondence should be addressed to Junhao Hu; junhaomath@163.com

Received 5 February 2015; Revised 2 May 2015; Accepted 2 May 2015

Academic Editor: Zidong Wang

Copyright (C) 2015 Chunmei Wu et al. This is an open access article distributed under the Creative Commons Attribution License, which permits unrestricted use, distribution, and reproduction in any medium, provided the original work is properly cited.

\begin{abstract}
We analyze the robustness of global exponential stability of hybrid stochastic neural networks subject to neutral terms and timevarying delays simultaneously. Given globally exponentially stable hybrid stochastic neural networks, we characterize the upper bounds of contraction coefficients of neutral terms and time-varying delays by using the transcendental equation. Moreover, we prove theoretically that, for any globally exponentially stable hybrid stochastic neural networks, if additive neutral terms and timevarying delays are smaller than the upper bounds arrived, then the perturbed neural networks are guaranteed to also be globally exponentially stable. Finally, a numerical simulation example is given to illustrate the presented criteria.
\end{abstract}

\section{Introduction}

In the past decades, neural networks have been extensively used in various areas. Stability is usually a prerequisite for most successful applications of neural networks. The stability of neural networks depends mainly on their parametric configuration. Moreover, it is well known that noise and time delays are often the sources of instability and they may destroy the stability of neural networks if they exceed their limits.

Robustness is the traditional characteristic of disturbance rejection, and it refers to control system in the feature or parameter perturbation when it still can make the performance of the quality indicators remain unchanged. Robustness characterized control system is not sensitive to feature or parameter perturbation. In practical problems, the system characteristics or parameter perturbation is often unavoidable. Causes of perturbation mainly have two aspects; one is due to the imprecision of the measurement features or the fact that parameters will deviate from the actual value of the design value (nominal value); the other is influenced by environmental factors in the process of system operation and causes the slow drift of features or parameters. Robustness, therefore, has become an important research topic in the control theory. The study of robustness is mainly limited to linear time-invariant control system; the areas covered include stability and astatic and adaptive control. Robustness problems and relative stability of the control system and the principle of invariance have the close relation; the establishment of the internal model principle is the study of the fact that robustness problem plays an important role.

The robustness analyses of global exponential stability of various neural networks have been widely investigated in recent years. For example, in [1], the robustness of global exponential stability for hybrid neural networks with noise and delay perturbations was investigated. In [2], the robustness of global exponential stability of recurrent neural networks with time delays and random disturbances was discussed. In [3], delay-dependent robust stability of cellular neural networks with discrete and distributed time-varying delays was considered. Shen and Wang [4] studied robustness of global exponential stability of recurrent neural networks in the presence of time delays and random disturbances. The stability of the systems often also depends on neutral terms. In [5], Shen and Wang continue to analyse robustness of global exponential stability of nonlinear systems with time delays and neutral terms. 
In implementation or application of neural networks, the change of parameters of neural networks abruptly is not uncommon. In [6], Liberzon characterizes the parameters of neural network (e.g., connection weights and biases) and reports that they change abruptly due to unexpected failure or designed switching. In such a case, neural networks can be represented by a switching model which can be regarded as a set of parametric configurations switching from one to another according to a given Markov chain. For instance, stability of stochastic neural networks with Markovian jumping Parameters was analyzed in [7]. In [8], stability analysis for discrete time Markovian jump neural networks with mixed time delays was studied. Stability of several hybrid stochastic neural networks was analyzed in [9-13]. In [14], the global asymptotic stability of cellular neural networks with delays was investigated. In $[15,16]$, the authors investigated the robustness of global exponential stability of stochastic systems (with Markovian switching) in the presence of time-varying delays or noises. In [17], the robust stability analysis of switched Hopfield neural networks with time-varying delay under uncertainty was investigated. In $[18,19]$, the delay-dependent robust stability of uncertain neutral-type stochastic systems with Markovian jumping was studied by using linear matrix inequality.

In this paper, we characterize robustness of the hybrid stochastic neural networks in the presence of neutral terms and time-varying delays. The upper bounds of neutral terms contraction coefficients and time-varying delays are estimated by solving transcendental equations. Moreover, we prove theoretically that, for any globally exponentially stable hybrid stochastic neural networks, if additive contraction coefficients of neutral terms and time-varying delays are smaller than the derived upper bounds herein, then the perturbed neural networks are guaranteed to be globally exponentially stable.

The remainder of this paper is organized as follows. In Section 2, some preliminaries and necessary notations are given. Section 3 discusses the stability conditions of the neural networks with Markovian switching in the presence of neutral terms and time-varying delays simultaneously. In Section 4, a numerical example is given to substantiate the theoretical results. Finally, concluding remarks are made in Section 5.

\section{Preliminaries and Assumptions}

Throughout this paper, unless otherwise specified, let $\left(\Omega, \mathscr{F},\left\{\mathscr{F}_{t}\right\}_{t \geq 0}, \mathbb{P}\right)$ be a complete probability space with a filtration $\left\{\mathscr{F}_{t}\right\}_{t \geq 0}$ satisfying the usual conditions (i.e., it is right continuous and $\mathscr{F}_{0}$ contains all $\mathbb{P}$-null sets). Let $w(t)=$ $\left(w_{1}(t), \ldots, w_{m}(t)\right)^{T}$, and let $t \geq 0$ be an $m$-dimension Brownian motion defined on the probability space. Let | $\cdot \mid$ be the Euclidean norm in $\mathbb{R}^{n}$. If $A$ is a vector or matrix, its transpose is denoted by $A^{T}$. If $A$ is a matrix, its trace norm is denoted by $|A|=\sqrt{\operatorname{trace}\left(A^{T} A\right)}$. Let $C\left([-\bar{\tau}, 0] ; \mathbb{R}^{n}\right)$ denote the family of continuous functions $\varphi$ from $[-\bar{\tau}, 0]$ to $\mathbb{R}^{n}$ with the norm $\|\varphi\|=\sup _{-\bar{\tau} \leq \theta \leq 0}|\varphi(\theta)|$.
For $p>0$, denote by $\mathscr{L}_{\mathscr{F}_{0}}^{p}\left([-\bar{\tau}, 0] ; \mathbb{R}^{n}\right)$ the family of all $\mathscr{F}_{0^{-}}$ measurable, $C\left([-\bar{\tau}, 0] ; \mathbb{R}^{n}\right)$-valued random variables $\xi$ such that $\mathbb{E}\|\xi\|^{p}<\infty$. Denote by $\mathscr{C}_{\mathscr{F}_{0}}^{b}\left([-\bar{\tau}, 0], \mathbb{R}^{n}\right)$ the family of all $\mathscr{F}_{0}$-measurable, bounded, and $C\left([-\bar{\tau}, 0] ; \mathbb{R}^{n}\right)$-valued random variables.

Let $r(t), t \geq 0$, be a right continuous Markov chain on the probability space taking values in a finite state space $S=$ $\{1,2, \ldots, m\}$ with generator $\Gamma=\left(\gamma_{i j}\right)_{m \times m}$ given by

$$
\begin{aligned}
& p\{r(t+\delta)=j \mid r(t)=i\} \\
& \quad= \begin{cases}\gamma_{i j} \delta+o(\delta): & \text { if } i \neq j, \\
1+\gamma_{i j} \delta+o(\delta): & \text { if } i=j,\end{cases}
\end{aligned}
$$

where $\delta>0$. Here, $\gamma_{i j} \geq 0$ is the transition rate from $i$ to $j$ if $i \neq j$, while $\gamma_{i i}=-\sum_{j \neq i} \gamma_{i j}$. It is known that almost every sample path of $r(t)$ is a right continuous step function with a finite number of simple jumps in any finite subinterval of $R_{+}$.

In this paper, we will consider the hybrid stochastic neural networks in the presence of neutral terms and time-varying delays of the form

$$
\begin{aligned}
& \mathrm{d}[y(t)-G(y(t-\tau(t)))] \\
& =A(r(t)) f(y(t), y(t-\tau(t)), t) \mathrm{d} t \\
& \quad+B(r(t)) g(y(t), y(t-\tau(t)), t) \mathrm{d} w(t), \\
& \quad \\
& \quad t \geq t_{0}
\end{aligned}
$$$$
y(t)=\psi\left(t-t_{0}\right), \quad t_{0}-\bar{\tau} \leq t \leq t_{0},
$$

where $f: \mathbb{R}^{n} \times \mathbb{R}^{n} \times \mathbb{R}_{+} \rightarrow \mathbb{R}^{n}, g: \mathbb{R}^{n} \times \mathbb{R}^{n} \times \mathbb{R}_{+} \rightarrow$ $\mathbb{R}^{n \times m}, G: \mathbb{R}^{n} \rightarrow \mathbb{R}^{n}, t_{0} \geq 0, A(i)=\operatorname{diag}\left\{a_{1}(i), \ldots, a_{n}(i)\right\} \in$ $R^{n \times n}$ is the self-feedback connection weight matrix, $B(i)=$ $\left(b_{k l}(i)\right)_{n \times n} \in R^{n \times n}$ is connection weight matrix, $w(t)$ is an $m$ dimension Brownian motion defined on the probability space $\left(\Omega, \mathscr{F},\left\{\mathscr{F}_{t}\right\}_{t \geq 0}, \mathbb{P}\right), \tau(t)$ is a time-varying delay, which satisfies $\tau(t):\left[t_{0},+\infty\right) \rightarrow[0, \bar{\tau}], \dot{\tau}(t) \leq \mu<1$, and $\psi=\{\psi(s):-\bar{\tau} \leq$ $s \leq 0\} \in \mathscr{C}_{\mathscr{F}_{0}}^{b}\left([-\bar{\tau}, 0], \mathbb{R}^{n}\right)$. Assume that $f, g$, and $G$ satisfy the following assumptions.

Assumption 1. For all $u, v, \bar{u}, \bar{v} \in \mathbb{R}^{n}$ and $t \in \mathbb{R}_{+}$, there exists a constant $K_{1}>0$ such that

$$
|f(u, \bar{u}, t)-f(v, \bar{v}, t)| \leq K_{1}(|u-v|+|\bar{u}-\bar{v}|) ;
$$

moreover, for any $t \in \mathbb{R}_{+}, f(0,0, t) \equiv 0$.

Assumption 2. For all $u, v, \bar{u}, \bar{v} \in \mathbb{R}^{n}$ and $t \in \mathbb{R}_{+}$, there exists a positive constant $K_{2}$ such that

$$
|g(u, \bar{u}, t)-g(v, \bar{v}, t)| \leq K_{2}(|u-v|+|\bar{u}-\bar{v}|) ;
$$

moreover, for any $t \in \mathbb{R}_{+}, g(0,0, t) \equiv 0$.

Assumption 3. For all $u, v \in \mathbb{R}^{n}$, there exists a constant $k \in$ $(0,1)$ such that

$$
|G(u)-G(v)| \leq k|u-v|
$$

moreover, $G(0)=0$. 
It is well known that, for any given initial value $t_{0}$ and $\psi$, according to Assumptions 1-3, system (2) has a unique state $y\left(t ; t_{0}, \psi\right)$ when $t \geq t_{0}-\bar{\tau}$ (see [20]). Besides, system (2) has a trivial state $y \equiv 0$.

In case of no time delay and neutral term, system (2) has the following form:

$$
\begin{aligned}
\mathrm{d} x(t)= & A(r(t)) f(x(t), x(t), t) \mathrm{d} t \\
& +B(r(t)) g(x(t), x(t), t) \mathrm{d} w(t), \quad t>t_{0} \\
x\left(t_{0}\right)= & \psi(0) \in \mathbb{R}^{n} .
\end{aligned}
$$

From [20], according to Assumptions 1-2, for any given initial value $t_{0}$ and $\psi(0)$, for system (6), there exists a unique state $x\left(t ; t_{0}, \psi(0)\right)$. And $x \equiv 0$ is the trivial state of system (6). To analyse the stability of systems (6) and (2), we now define the $p$ th moment global exponential stability and almost surely global exponential stability of systems (6) and (2).

Definition 4. Let $p \geq 2$; if for any $t_{0} \in \mathbb{R}_{+}$and $\psi(0) \in \mathbb{R}^{n}$, there exist positive constants $\alpha$ and $\beta$ such that

$$
\begin{aligned}
& \mathbb{E}\left|x\left(t ; t_{0}, \psi(0)\right)\right|^{p} \leq \alpha|\psi(0)| \exp \left(-\beta\left(t-t_{0}\right)\right), \\
& t \geq t_{0} \text {, }
\end{aligned}
$$

where $x\left(t ; t_{0}, \psi(0)\right)$ is the state of system (6); then the state of system (6) is pth moment globally exponentially stable.

The state of system (2) is pth moment globally exponentially stable if, for any $t_{0} \in \mathbb{R}_{+}, \psi \in \mathscr{C}_{\mathscr{F}_{0}}^{b}\left([-\tau, 0] ; \mathbb{R}^{n}\right)$, there exist $\widetilde{\alpha}>0$ and $\widetilde{\beta}>0$ such that

$$
\mathbb{E}\left|y\left(t ; t_{0}, \psi\right)\right|^{p} \leq \widetilde{\alpha}\|\psi\| \exp \left(-\widetilde{\beta}\left(t-t_{0}\right)\right), \quad t \geq t_{0},
$$

that is, the Lyapunov exponent

$$
\limsup _{t \rightarrow \infty}\left(\frac{\ln \left(\mathbb{E}\left|y\left(t ; t_{0}, \psi\right)\right|^{p}\right)}{t}\right)<0,
$$

where $y\left(t ; t_{0}, \psi\right)$ is the state of system (2).

Definition 5. System (6) is said to be almost surely globally exponentially stable, if, for any $t_{0} \in \mathbb{R}_{+}$and $\psi(0) \in \mathbb{R}^{n}$, there exist $\alpha>0$ and $\beta>0$ such that $\left|x\left(t ; t_{0}, \psi(0)\right)\right| \leq$ $\alpha|\psi(0)| \exp \left(-\beta\left(t-t_{0}\right)\right), t \geq t_{0}$ holds almost surely, where $x\left(t ; t_{0}, \psi(0)\right)$ is the state of system (6).

If, for any $t_{0} \in \mathbb{R}_{+}$and $\psi(0) \in \mathbb{R}^{n}$, there exist $\widetilde{\alpha}>0$ and $\widetilde{\beta}>0$ such that $\left|y\left(t ; t_{0}, \psi\right)\right| \leq \widetilde{\alpha}\|\psi\| \exp \left(-\widetilde{\beta}\left(t-t_{0}\right)\right), t \geq t_{0}$ holds almost surely; then system (2) is almost surely globally exponentially stable, where $y\left(t ; t_{0}, \psi\right)$ is the state of system (2).

From the above definitions, it is clear that the almost sure global exponential stability of system (2) implies the $p$ th moment global exponential stability of system (2) (see [20]) but not vice versa. However, if Assumptions 1-3 hold, we can get the following lemma (see [20]).

Lemma 6. Let Assumptions 1-3 hold. The pth moment global exponential stability of system (2) implies the almost surely global exponential stability.
Meanwhile, in order to obtain our result, we also need another lemma (see [20]).

Lemma 7. Let $p \geq 2$ and $h: \mathbb{R}^{n} \times \mathbb{R}^{n} \times\left[t_{0}, t\right] \rightarrow \mathbb{R}^{n \times m}$, such that

$$
\mathbb{E} \int_{t_{0}}^{t}|h(s)|^{p} \mathrm{~d} s<\infty
$$

then

$$
\begin{aligned}
& \mathbb{E}\left|\int_{t_{0}}^{t} h(s) \mathrm{d} w(s)\right|^{p} \\
& \quad \leq\left(\frac{p(p-1)}{2}\right)^{p / 2}\left(t-t_{0}\right)^{(p-2) / 2} \mathbb{E} \int_{t_{0}}^{t}|h(s)|^{p} \mathrm{~d} s .
\end{aligned}
$$

In particular, for $p=2$, there is equality.

\section{Robustness Analysis}

In this section, we will show that if system (6) is pth moment globally exponentially stable, system (2) may remain to be $p$ th moment globally exponentially stable provided that neutral terms contraction coefficients $k$ and time-varying delay $\bar{\tau}$ are sufficiently small.

Theorem 8. Let Assumptions 1-3 hold and system (6) is pth moment globally exponentially stable. System (2) is pth moment globally exponentially stable and almost surely globally exponentially stable, if $k<\min \left\{\tilde{k}, \exp \left(-2 \Delta\left(4^{p-1}+\right.\right.\right.$ $\left.\left.\left.2^{p-1}\right) c_{1}\right) /\left(2^{p-1} c_{1} \Delta 12^{p-1} 2^{p}(1 /(1-\mu)+1 /(1-2 \mu))+8^{p-1} /(1-\mu)\right)\right\}$ and $\bar{\tau}<\min \{\Delta / 2, \widetilde{\tau}\}$, where $2 \widetilde{k}$ is a unique positive solution of the transcendental equation

$$
\begin{aligned}
& {\left[\Delta 12^{p-1}(4 k)^{p}\left(\frac{1}{1-\mu}+\frac{1}{1-2 \mu}\right) c_{1}\right.} \\
& \left.\quad+8^{p-1} k^{p}\left(1+\frac{3}{1-\mu}\right)\right] \exp \left(2 \Delta c_{1}\left(2^{p-1}+4^{p-1}\right)\right) \\
& +2^{p-1} \alpha^{p} \exp (-p \beta(\Delta-\bar{\tau}))=1,
\end{aligned}
$$

and $\tilde{\tau}$ is a unique positive solution of the transcendental equation

$$
\begin{aligned}
& {\left[\left[\Delta 12^{p-1}(4 k)^{p}\left(\frac{1}{1-\mu}+\frac{1}{1-2 \mu}\right)\right.\right.} \\
& \left.\quad+16^{p-1} \bar{\tau}\left(1+\frac{1}{1-\mu}\right)\right] c_{1}+8^{p-1} c_{1} c_{2}+16^{p-1} \\
& \left.\quad \cdot \frac{c_{1} c_{3}}{p \beta}(\alpha)^{p}+8^{p-1} k^{p}\left(1+\frac{3}{1-\mu}\right)\right] \\
& \quad \cdot \exp \left(2 \Delta c_{1}\left[\left(2^{p-1}+4^{p-1}\right)+c_{3} 8^{p-1}\right]\right)+2^{p-1} \alpha^{p} \\
& \quad \cdot \exp (-p \beta(\Delta-\bar{\tau}))=1,
\end{aligned}
$$


where

$$
\begin{aligned}
c_{1}= & \left(\frac{4 \Delta}{\varepsilon}\right)^{p-1}\left\|\bar{A}_{i}\right\|^{p} K_{1}^{p}+[4 \Delta p(p-1)]^{p / 2}\left\|\bar{B}_{i}\right\|^{p} \\
& \cdot \frac{K_{2}^{p}}{4 \Delta(1-\varepsilon)^{p-1}}, \\
c_{2}= & 6^{p-1} k^{p} \bar{\tau}\left(\frac{1}{1-\mu}+\frac{2}{1-2 \mu}\right)+(6 \bar{\tau})^{p-1}\left\|\bar{A}_{i}\right\|^{p} K_{1}^{p} \\
& \cdot \frac{\bar{\tau}^{2}}{1-\mu}+\frac{1}{6}[2 p(p-1) \bar{\tau}]^{p / 2}\left\|\bar{B}_{i}\right\|^{p}\left(3 K_{2}\right)^{p} \frac{\bar{\tau}}{1-\mu}, \\
c_{3}= & {\left[(6 \bar{\tau})^{p-1} \bar{\tau}\left\|\bar{A}_{i}\right\|^{p} K_{1}^{p}\right.} \\
& \left.+\frac{1}{6}[2 p(p-1) \bar{\tau}]^{p / 2}\left\|\bar{B}_{i}\right\|^{p}\left(3 K_{2}\right)^{p}\right]\left(1+\frac{1}{1-\mu}\right),
\end{aligned}
$$

and $\varepsilon$ is an adjustable parameter, $\varepsilon \in(0,1), \Delta$ is a step, and $\Delta-$ $\bar{\tau}>\ln \left(2^{p-1} \alpha^{p}\right) /(p \beta)>0,\left\|\bar{A}_{i}\right\|^{p}=\max _{i \in S}\|A(i)\|^{p},\left\|\bar{B}_{i}\right\|^{p}=$ $\max _{i \in S}\|B(i)\|^{p}$.

Proof. Fix $t_{0}, \psi=\{\psi(t),-\bar{\tau} \leq t \leq 0\}$, to simplicity, we write $x\left(t ; t_{0}, \psi(0)\right)$ and $y\left(t ; t_{0}, \psi\right)$ as $x(t)$ and $y(t)$, respectively. From systems (2) and (6), for any $t \geq t_{0}$, we have

$$
\begin{aligned}
& x(t)-y(t)+G(y(t-\tau(t)))-G\left(y\left(t_{0}-\tau\left(t_{0}\right)\right)\right) \\
& =A(i) \int_{t_{0}}^{t}[f(x(s), x(s), s) \\
& \quad-f(y(s), y(s-\tau(s)), s)] \mathrm{d} s+B(i) \\
& \quad \cdot \int_{t_{0}}^{t}[g(x(s), x(s), s)-g(y(s), y(s-\tau(s)), s)] \mathrm{d} w(s),
\end{aligned}
$$

when $t \leq t_{0}+2 \Delta$; by Assumptions 1-2, Hölder inequality, and Lemma 7 , we derive

$$
\begin{aligned}
& \mathbb{E} \mid x(t)-y(t)+G(y(t-\tau(t)))-G\left(y \left(t_{0}\right.\right. \\
& \left.\left.-\tau\left(t_{0}\right)\right)\right)\left.\right|^{p} \leq\left.\frac{1}{\varepsilon^{p-1}}\left\|\overline{A_{i}}\right\|\right|^{p} \mathbb{E} \mid \int_{t_{0}}^{t}[f(x(s), x(s), s) \\
& -f(y(s), y(s-\tau(s)), s)]\left.\mathrm{d} s\right|^{p}+\frac{1}{(1-\varepsilon)^{p-1}}\left\|\bar{B}_{i}\right\|^{p} \\
& \cdot \mathbb{E} \mid \int_{t_{0}}^{t}[g(x(s), x(s), s) \\
& -g(y(s), y(s-\tau(s)), s)]\left.\mathrm{d} w(s)\right|^{p} \leq\left(\frac{2 \Delta}{\varepsilon}\right)^{p-1} \\
& \cdot\left\|\bar{A}_{i}\right\|^{p} \int_{t_{0}}^{t} \mathbb{E} \mid f(x(s), x(s), s) \\
& -\left.f(y(s), y(s-\tau(s)), s)\right|^{p} \mathrm{~d} s+\frac{1}{(1-\varepsilon)^{p-1}}\left\|\bar{B}_{i}\right\|^{p}
\end{aligned}
$$

$$
\begin{aligned}
& \cdot\left[\frac{p(p-1)}{2}\right]^{p / 2}(2 \Delta)^{(p-2) / 2} \int_{t_{0}}^{t} \mathbb{E} \mid g(x(s), x(s), s) \\
& -\left.g(y(s), y(s-\tau(s)), s)\right|^{p} \mathrm{~d} s \leq\left(\frac{4 \Delta}{\varepsilon}\right)^{p-1}\left\|\bar{A}_{i}\right\|^{p} \\
& \cdot K_{1}^{p}\left[\int_{t_{0}}^{t} \mathbb{E}|x(s)-y(s)|^{p} \mathrm{~d} s+\int_{t_{0}}^{t} \mathbb{E} \mid x(s)\right. \\
& \left.-\left.y(s-\tau(s))\right|^{p} \mathrm{~d} s\right]+\frac{1}{4 \Delta}[4 \Delta p(p-1)]^{p / 2}\left\|\bar{B}_{i}\right\|^{p} \\
& \cdot \frac{K_{2}^{p}}{(1-\varepsilon)^{p-1}}\left[\int_{t_{0}}^{t} \mathbb{E}|x(s)-y(s)|^{p} \mathrm{~d} s+\int_{t_{0}}^{t} \mathbb{E} \mid x(s)\right. \\
& \left.-\left.y(s-\tau(s))\right|^{p} \mathrm{~d} s\right]:=c_{1} \int_{t_{0}}^{t} \mathbb{E}|x(s)-y(s)|^{p} \mathrm{~d} s \\
& +c_{1} \int_{t_{0}}^{t} \mathbb{E}|x(s)-y(s)+y(s)-y(s-\tau(s))|^{p} \mathrm{~d} s \\
& \leq\left(2^{p-1}+1\right) c_{1} \int_{t_{0}}^{t} \mathbb{E}|x(s)-y(s)|^{p} \mathrm{~d} s \\
& +2^{p-1} c_{1} \int_{t_{0}}^{t} \mathbb{E}|y(s)-y(s-\tau(s))|^{p} \mathrm{~d} s,
\end{aligned}
$$

where $c_{1}=(4 \Delta / \varepsilon)^{p-1}\left\|\bar{A}_{i}\right\|^{p} K_{1}^{p}+[4 \Delta p(p-1)]^{p / 2}\left\|\bar{B}_{i}\right\|^{p} K_{2}^{p} /$ $\left(4 \Delta(1-\varepsilon)^{p-1}\right)$.

By system (2) and Assumptions 1-3, we get

$$
\begin{aligned}
& \mathbb{E}|y(s)-y(s-\tau(s))|^{p} \\
& \leq 3^{p-1} \mathbb{E}|G(y(s-\tau(s)))-G(y(s-2 \tau(s)))|^{p} \\
& +3^{p-1}\left\|\bar{A}_{i}\right\|^{p} \int_{s-\bar{\tau}}^{s} \mathbb{E}|f(y(r), y(r-\tau(r)), r) \mathrm{d} r|^{p} \\
& +3^{p-1}\left\|\bar{B}_{i}\right\|^{p} \\
& \cdot \mathbb{E}\left|\int_{s-\bar{\tau}}^{s} g(y(r), y(r-\tau(r)), r) \mathrm{d} w(r)\right|^{p} \\
& \leq 3^{p-1} k^{p} \mathbb{E}|y(s-\tau(s))-y(s-2 \tau(s))|^{p} \\
& +(3 \bar{\tau})^{p-1}\left\|\bar{A}_{i}\right\|^{p} \\
& \cdot \int_{s-\bar{\tau}}^{s} \mathbb{E}|f(y(r), y(r-\tau(r)), r)|^{p} \mathrm{~d} r \\
& +3^{p-1}\left[\frac{p(p-1)}{2}\right]^{p / 2}(\bar{\tau})^{(p-2) / 2}\left\|\bar{B}_{i}\right\|^{p} \\
& \cdot \mathbb{E} \int_{s-\bar{\tau}}^{s}|g(y(r), y(r-\tau(r)), r)|^{p} \mathrm{~d} r \\
& \leq 3^{p-1} k^{p} \mathbb{E}|y(s-\tau(s))-y(s-2 \tau(s))|^{p} \\
& +(6 \bar{\tau})^{p-1}\left\|\bar{A}_{i}\right\|^{p}
\end{aligned}
$$


Discrete Dynamics in Nature and Society

5

$$
\begin{aligned}
& \cdot K_{1}^{p}\left[\int_{s-\bar{\tau}}^{s} \mathbb{E}|y(r)|^{p} \mathrm{~d} r+\int_{s-\bar{\tau}}^{s} \mathbb{E}|y(r-\tau(r))|^{p} \mathrm{~d} r\right] \\
& +\frac{1}{6 \bar{\tau}}[2 \bar{\tau} p(p-1)]^{p / 2}\left\|\bar{B}_{i}\right\|^{p}\left(3 K_{2}\right)^{p} \\
& \cdot\left[\int_{s-\bar{\tau}}^{s} \mathbb{E}|y(r)|^{p} \mathrm{~d} r+\int_{s-\bar{\tau}}^{s} \mathbb{E}|y(r-\tau(r))|^{p} \mathrm{~d} r\right] .
\end{aligned}
$$

In addition, for $t_{0}+\tau \leq t \leq t_{0}+2 \Delta$,

$$
\begin{gathered}
3^{p-1} k^{p} \int_{t_{0}+\bar{\tau}}^{t} \mathbb{E}|y(s-\tau(s))-y(s-2 \tau(s))|^{p} \mathrm{~d} s \\
\leq 6^{p-1} k^{p} \int_{t_{0}+\bar{\tau}}^{t} \mathbb{E}|y(s-\tau(s))|^{p} \mathrm{~d} s
\end{gathered}
$$$$
+6^{p-1} k^{p} \int_{t_{0}+\bar{\tau}}^{t} \mathbb{E}|y(s-2 \tau(s))|^{p} \mathrm{~d} s \leq 6^{p-1} k^{p} \frac{1}{1-\mu}
$$$$
\cdot \int_{t_{0}}^{t-\bar{\tau}} \mathbb{E}|y(s)|^{p} \mathrm{~d} s+6^{p-1} k^{p} \frac{1}{1-2 \mu}
$$$$
\cdot \int_{t_{0}-\bar{\tau}}^{t-2 \bar{\tau}} \mathbb{E}|y(s)|^{p} \mathrm{~d} s \leq 6^{p-1} k^{p} \frac{1}{1-\mu}
$$$$
\cdot \int_{t_{0}}^{t-\bar{\tau}} \mathbb{E}|y(s)|^{p} \mathrm{~d} s+6^{p-1} k^{p} \frac{1}{1-2 \mu}
$$$$
\cdot \int_{t_{0}-\bar{\tau}}^{t_{0}} \mathbb{E}|y(s)|^{p} \mathrm{~d} s+6^{p-1} k^{p} \frac{1}{1-2 \mu}
$$$$
\cdot \int_{t_{0}}^{t-\bar{\tau}} \mathbb{E}|y(s)|^{p} \mathrm{~d} s \leq 6^{p-1} k^{p}
$$$$
\begin{aligned}
& \cdot \frac{\bar{\tau}}{1-2 \mu}\left(\sup _{t_{0}-\bar{\tau} \leq s \leq t_{0}} \mathbb{E}|y(s)|^{p}\right) \\
& +6^{p-1} k^{p}\left(\frac{1}{1-\mu}+\frac{1}{1-2 \mu}\right) \int_{t_{0}}^{t-\bar{\tau}} \mathbb{E}|y(s)|^{p} \mathrm{~d} s
\end{aligned}
$$$$
\leq 6^{p-1} k^{p} \frac{\bar{\tau}}{1-2 \mu}\left(\sup _{t_{0}-\bar{\tau} \leq s \leq t_{0}} \mathbb{E}|y(s)|^{p}\right)
$$$$
+6^{p-1} k^{p}\left(\frac{1}{1-\mu}+\frac{1}{1-2 \mu}\right) \int_{t_{0}}^{t_{0}+\bar{\tau}} \mathbb{E}|y(s)|^{p} \mathrm{~d} s
$$$$
+6^{p-1} k^{p}\left(\frac{1}{1-\mu}+\frac{1}{1-2 \mu}\right) \int_{t_{0}+\bar{\tau}}^{t_{0}-\bar{\tau}+2 \Delta} \mathbb{E}|y(s)|^{p} \mathrm{~d} s
$$$$
\leq 6^{p-1} k^{p} \frac{\bar{\tau}}{1-2 \mu}\left(\sup _{t_{0}-\bar{\tau} \leq s \leq t_{0}} \mathbb{E}|y(s)|^{p}\right)
$$$$
+6^{p-1} k^{p} \bar{\tau}\left(\frac{1}{1-\mu}+\frac{1}{1-2 \mu}\right)\left(\sup _{t_{0} \leq s \leq t_{0}+\bar{\tau}} \mathbb{E}|y(s)|^{p}\right)
$$$$
+(2 \Delta-2 \bar{\tau}) 6^{p-1} k^{p}\left(\frac{1}{1-\mu}+\frac{1}{1-2 \mu}\right)
$$

$$
\begin{aligned}
& \cdot\left(\sup _{t_{0}+\bar{\tau} \leq s \leq t_{0}-\bar{\tau}+2 \Delta} \mathbb{E}|y(s)|^{p}\right) \\
& \leq 6^{p-1} k^{p} \bar{\tau}\left(\frac{1}{1-\mu}+\frac{2}{1-2 \mu}\right) \\
& \cdot\left(\sup _{t_{0}-\bar{\tau} \leq s \leq t_{0}+\bar{\tau}} \mathbb{E}|y(s)|^{p}\right) \\
& +2 \Delta 6^{p-1} k^{p}\left(\frac{1}{1-\mu}+\frac{1}{1-2 \mu}\right) \\
& \cdot\left(\sup _{t_{0}+\bar{\tau} \leq s \leq t_{0}-\bar{\tau}+2 \Delta} \mathbb{E}|y(s)|^{p}\right) .
\end{aligned}
$$

By reversing the order of integral, we get

$$
\begin{aligned}
& \int_{t_{0}+\bar{\tau}}^{t} \mathrm{~d} s \int_{s-\bar{\tau}}^{s} \mathbb{E}|y(r)|^{p} \mathrm{~d} r \\
& \quad=\int_{t_{0}}^{t} \mathrm{~d} r \int_{\max \left(t_{0}+\bar{\tau}, r\right)}^{\min (r+\bar{\tau}, t)} \mathbb{E}|y(r)|^{p} \mathrm{~d} s \\
& \leq \bar{\tau} \int_{t_{0}}^{t} \mathbb{E}|y(r)|^{p} \mathrm{~d} r .
\end{aligned}
$$

Similarly, we can derive

$$
\begin{aligned}
& \int_{t_{0}+\bar{\tau}}^{t} \mathrm{~d} s \int_{s-\bar{\tau}}^{s} \mathbb{E}|y(r-\tau(r))|^{p} \mathrm{~d} r \\
& =\int_{t_{0}}^{t} \mathrm{~d} r \int_{\max \left(t_{0}+\bar{\tau}, r\right)}^{\min (r+\bar{\tau}, t)} \mathbb{E}|y(r-\tau(r))|^{p} \mathrm{~d} s \\
& \leq \bar{\tau} \int_{t_{0}}^{t} \mathbb{E}|y(r-\tau(r))|^{p} \mathrm{~d} r \\
& \leq \frac{\bar{\tau}}{1-\mu} \int_{t_{0}-\bar{\tau}}^{t} \mathbb{E}|y(u)|^{p} \mathrm{~d} u \\
& \leq \frac{\bar{\tau}^{2}}{1-\mu}\left(\sup _{t_{0}-\bar{\tau} \leq s \leq t_{0}} \mathbb{E}|y(s)|^{p}\right) \\
& +\frac{\bar{\tau}}{1-\mu} \int_{t_{0}}^{t} \mathbb{E}|y(r)|^{p} \mathrm{~d} r .
\end{aligned}
$$

Therefore, when $t \geq t_{0}+\bar{\tau}$, by substituting (18)-(20) into (17), we have

$$
\begin{aligned}
& \int_{t_{0}+\bar{\tau}}^{t} \mathbb{E}|y(s)-y(s-\tau(s))|^{p} \mathrm{~d} s \leq 6^{p-1} k^{p} \bar{\tau}\left(\frac{1}{1-\mu}\right. \\
& \left.+\frac{2}{1-2 \mu}\right)\left(\sup _{t_{0}-\bar{\tau} \leq s \leq t_{0}+\bar{\tau}} \mathbb{E}|y(s)|^{p}\right) \\
& +\left[(6 \bar{\tau})^{p-1}\left\|\bar{A}_{i}\right\|^{p} K_{1}^{p} \frac{\bar{\tau}^{2}}{1-\mu}\right.
\end{aligned}
$$


6

Discrete Dynamics in Nature and Society

$$
\begin{aligned}
& \left.+\frac{1}{6}[2 p(p-1) \bar{\tau}]^{p / 2}\left\|\bar{B}_{i}\right\|^{p}\left(3 K_{2}\right)^{p} \frac{\bar{\tau}}{1-\mu}\right] \\
& \cdot\left(\sup _{t_{0}-\bar{\tau} \leq s \leq t_{0}+\bar{\tau}} \mathbb{E}|y(s)|^{p}\right)+2 \Delta 6^{p-1} k^{p}\left(\frac{1}{1-\mu}\right. \\
& \left.+\frac{1}{1-2 \mu}\right)\left(\sup _{t_{0}+\bar{\tau} \leq s \leq t_{0}-\bar{\tau}+2 \Delta} \mathbb{E}|y(s)|^{p}\right) \\
& +\left[(6 \bar{\tau})^{p-1} \bar{\tau} \| \bar{A}_{i}||^{p} K_{1}^{p}\right. \\
& \left.+\frac{1}{6}[2 p(p-1) \bar{\tau}]^{p / 2}\left\|\bar{B}_{i}\right\|^{p}\left(3 K_{2}\right)^{p}\right]\left(1+\frac{1}{1-\mu}\right) \\
& \cdot \int_{t_{0}}^{t} \mathbb{E}|y(r)|^{p} \mathrm{~d} r:=c_{2}\left(\sup _{t_{0}-\bar{\tau} \leq s \leq t_{0}+\bar{\tau}} \mathbb{E}|y(s)|^{p}\right) \\
& +c_{3} \int_{t_{0}}^{t} \mathbb{E}|y(r)|^{p} \mathrm{~d} r+2 \Delta 6^{p-1} k^{p}\left(\frac{1}{1-\mu}+\frac{1}{1-2 \mu}\right) \\
& \cdot\left(\sup _{t_{0}+\bar{\tau} \leq s \leq t_{0}-\bar{\tau}+2 \Delta} \mathbb{E}|y(s)|^{p}\right),
\end{aligned}
$$

(21)

where

$$
\begin{aligned}
c_{2} & =6^{p-1} k^{p} \bar{\tau}\left(\frac{1}{1-\mu}+\frac{2}{1-2 \mu}\right)+(6 \bar{\tau})^{p-1}\left\|\bar{A}_{i}\right\|^{p} K_{1}^{p} \\
& \cdot \frac{\bar{\tau}^{2}}{1-\mu}+\frac{1}{6}[2 p(p-1) \bar{\tau}]^{p / 2}\left\|\bar{B}_{i}\right\|^{p}\left(3 K_{2}\right)^{p} \frac{\bar{\tau}}{1-\mu}, \\
c_{3} & =\left[(6 \bar{\tau})^{p-1} \bar{\tau}\left\|\bar{A}_{i}\right\|^{p} K_{1}^{p}\right. \\
& \left.+\frac{1}{6}[2 p(p-1) \bar{\tau}]^{p / 2}\left\|\bar{B}_{i}\right\|^{p}\left(3 K_{2}\right)^{p}\right]\left(1+\frac{1}{1-\mu}\right) .
\end{aligned}
$$

From (21) and (16), when $t \leq t_{0}+2 \Delta$, we get

$$
\begin{aligned}
& \mathbb{E} \mid x(t)-y(t)+G(y(t-\tau(t))) \\
& -\left.G\left(y\left(t_{0}-\tau\left(t_{0}\right)\right)\right)\right|^{p} \leq\left(2^{p-1}+1\right) \\
& \cdot c_{1} \int_{t_{0}}^{t} \mathbb{E}|x(s)-y(s)|^{p} \mathrm{~d} s \\
& +2^{p-1} c_{1} \int_{t_{0}}^{t_{0}+\bar{\tau}} \mathbb{E}|y(s)-y(s-\tau(s))|^{p} \mathrm{~d} s \\
& +2^{p-1} c_{1} \int_{t_{0}+\bar{\tau}}^{t} \mathbb{E}|y(s)-y(s-\tau(s))|^{p} \mathrm{~d} s \leq\left(2^{p-1}\right. \\
& +1) c_{1} \int_{t_{0}}^{t} \mathbb{E}|x(s)-y(s)|^{p} \mathrm{~d} s+4^{p-1} c_{1} \bar{\tau}(1 \\
& \left.+\frac{1}{1-\mu}\right)\left(\sup _{t_{0}-\bar{\tau} \leq s \leq t_{0}+\bar{\tau}} \mathbb{E}|y(s)|^{p}\right)
\end{aligned}
$$

$$
\begin{aligned}
& +2^{p-1} c_{1} c_{2}\left(\sup _{t_{0}-\bar{\tau} \leq s \leq t_{0}+\bar{\tau}} \mathbb{E}|y(s)|^{p}\right) \\
& +2^{p-1} c_{1} c_{3} \int_{t_{0}}^{t} \mathbb{E}|y(s)-x(s)+x(s)|^{p} \mathrm{~d} s \\
& +c_{1} \Delta 6^{p-1}(2 k)^{p}\left(\frac{1}{1-\mu}+\frac{1}{1-2 \mu}\right) \\
& \cdot\left(\sup _{t_{0}+\bar{\tau} \leq s \leq t_{0}-\bar{\tau}+2 \Delta} \mathbb{E}|y(s)|^{p}\right) \leq\left(2^{p-1}+1\right) \\
& \cdot c_{1} \int_{t_{0}}^{t} \mathbb{E}|x(s)-y(s)|^{p} \mathrm{~d} s+4^{p-1} c_{1} \bar{\tau}\left(1+\frac{1}{1-\mu}\right) \\
& \cdot\left(\sup _{t_{0}-\bar{\tau} \leq s \leq t_{0}+\bar{\tau}} \mathbb{E}|y(s)|^{p}\right) \\
& +2^{p-1} c_{1} c_{2}\left(\sup _{t_{0}-\bar{\tau} \leq s \leq t_{0}+\bar{\tau}} \mathbb{E}|y(s)|^{p}\right) \\
& +4^{p-1} c_{1} c_{3} \int_{t_{0}}^{t} \mathbb{E}|x(s)-y(s)|^{p} \mathrm{~d} s+c_{1} \Delta 6^{p-1}(2 k)^{p}
\end{aligned}
$$$$
\cdot\left(\frac{1}{1-\mu}+\frac{1}{1-2 \mu}\right)
$$

$$
\begin{aligned}
& \cdot\left(\sup _{t_{0}+\bar{\tau} \leq s \leq t_{0}-\bar{\tau}+2 \Delta} \mathbb{E}|y(s)|^{p}\right)+\frac{c_{1} c_{3}}{4 p \beta}(4 \alpha)^{p} \\
& \cdot\left(\sup _{t_{0}-\bar{\tau} \leq s \leq t_{0}} \mathbb{E}|y(s)|^{p}\right) \leq\left[\left(2^{p-1}+1\right) c_{1}+4^{p-1} c_{1} c_{3}\right] \\
& \cdot \int_{t_{0}}^{t} \mathbb{E}|x(s)-y(s)|^{p} \mathrm{~d} s+c_{1} \Delta 6^{p-1}(2 k)^{p}\left(\frac{1}{1-\mu}\right. \\
& \left.+\frac{1}{1-2 \mu}\right)\left(\sup _{t_{0}+\bar{\tau} \leq s \leq t_{0}-\bar{\tau}+2 \Delta} \mathbb{E}|y(s)|^{p}\right)+\left[2^{p-1} c_{1} c_{2}\right. \\
& \left.+\frac{c_{1} \mathcal{C}_{3}}{4 p \beta}(4 \alpha)^{p}+4^{p-1} c_{1} \bar{\tau}\left(1+\frac{1}{1-\mu}\right)\right] \\
& \cdot\left(\sup _{t_{0}-\bar{\tau} \leq s \leq t_{0}+\bar{\tau}} \mathbb{E}|y(s)|^{p}\right) .
\end{aligned}
$$

Note that $\Delta \geq 2 \bar{\tau}$; when $t_{0}+\bar{\tau} \leq t \leq t_{0}+2 \Delta$, by Assumption 3, we have

$$
\begin{aligned}
\mathbb{E}\left|G(y(t-\tau(t)))-G\left(y\left(t_{0}-\tau\left(t_{0}\right)\right)\right)\right|^{p} \\
\quad \leq k^{p} \mathbb{E}\left|y(t-\tau(t))-y\left(t_{0}-\tau\left(t_{0}\right)\right)\right|^{p} \\
\leq 2^{p-1} k^{p}\left(\sup _{t_{0}-\bar{\tau} \leq s \leq t_{0}+\bar{\tau}} \mathbb{E}|y(s)|^{p}\right)
\end{aligned}
$$


Discrete Dynamics in Nature and Society

7

$$
\begin{aligned}
& +2^{p-1} k^{p}\left(\sup _{t_{0}+\bar{\tau} \leq s \leq t_{0}+2 \Delta} \mathbb{E}|y(s-\tau(s))|^{p}\right) \\
\leq & 2^{p-1} k^{p}\left(\sup _{t_{0}-\bar{\tau} \leq s \leq t_{0}+\bar{\tau}} \mathbb{E}|y(s)|^{p}\right) \\
& +\frac{2^{p-1} k^{p}}{1-\mu}\left(\sup _{t_{0} \leq s \leq t_{0}-\bar{\tau}+2 \Delta} \mathbb{E}|y(s)|^{p}\right) \\
\leq & 2^{p-1} k^{p}\left(1+\frac{1}{1-\mu}\right)\left(\sup _{t_{0}-\bar{\tau} \leq s \leq t_{0}+\bar{\tau}} \mathbb{E}|y(s)|^{p}\right) \\
& +\frac{2^{p-1} k^{p}}{1-\mu}\left(\sup _{t_{0}+\bar{\tau} \leq s \leq t_{0}-\bar{\tau}+2 \Delta} \mathbb{E}|y(s)|^{p}\right) .
\end{aligned}
$$

By (23) and (24), we further have

$$
\begin{aligned}
& \mathbb{E}|x(t)-y(t)|^{p} \leq 2^{p-1} \mathbb{E} \mid x(t)-y(t) \\
& +G(y(t-\tau(t)))-\left.G\left(y\left(t_{0}-\tau\left(t_{0}\right)\right)\right)\right|^{p} \\
& +2^{p-1} \mathbb{E}\left|G(y(t-\tau(t)))-G\left(y\left(t_{0}-\tau\left(t_{0}\right)\right)\right)\right|^{p} \\
& \leq\left[\left(4^{p-1}+2^{p-1}\right) c_{1}+8^{p-1} c_{1} c_{3}\right] \\
& \cdot \int_{t_{0}}^{t} \mathbb{E}|x(s)-y(s)|^{p} \mathrm{~d} s+c_{1} \Delta 12^{p-1}(2 k)^{p}\left(\frac{1}{1-\mu}\right. \\
& \left.+\frac{1}{1-2 \mu}\right)\left(\sup _{t_{0}+\bar{\tau} \leq s \leq t_{0}-\bar{\tau}+2 \Delta} \mathbb{E}|y(s)|^{p}\right)+\left[4^{p-1} c_{1} c_{2}\right. \\
& \left.+\frac{c_{1} c_{3}}{8 p \beta}(8 \alpha)^{p}+8^{p-1} c_{1} \bar{\tau}\left(1+\frac{1}{1-\mu}\right)\right] \\
& \cdot\left(\sup _{t_{0}-\bar{\tau} \leq s \leq t_{0}+\bar{\tau}} \mathbb{E}|y(s)|^{p}\right)+4^{p-1} k^{p}\left(1+\frac{1}{1-\mu}\right) \\
& \cdot\left(\sup _{t_{0}-\bar{\tau} \leq s \leq t_{0}+\bar{\tau}} \mathbb{E}|y(s)|^{p}\right) \\
& +\frac{4^{p-1} k^{p}}{1-\mu}\left(\sup _{t_{0}+\bar{\tau} \leq s \leq t_{0}-\bar{\tau}+2 \Delta} \mathbb{E}|y(s)|^{p}\right) \\
& \leq\left[\left(4^{p-1}+2^{p-1}\right) c_{1}+8^{p-1} c_{1} c_{3}\right] \\
& \cdot \int_{t_{0}}^{t} \mathbb{E}|x(s)-y(s)|^{p} \mathrm{~d} s \\
& +\left[c_{1} \Delta 12^{p-1}(2 k)^{p}\left(\frac{1}{1-\mu}+\frac{1}{1-2 \mu}\right)+\frac{4^{p-1} k^{p}}{1-\mu}\right] \\
& \cdot\left(\sup _{t_{0}+\bar{\tau} \leq s \leq t_{0}-\bar{\tau}+2 \Delta} \mathbb{E}|y(s)|^{p}\right)+\left[4^{p-1} c_{1} c_{2}\right.
\end{aligned}
$$

$$
\begin{aligned}
& \left.+\frac{c_{1} c_{3}}{8 p \beta}(8 \alpha)^{p}+\left(8^{p-1} c_{1} \bar{\tau}+4^{p-1} k^{p}\right)\left(1+\frac{1}{1-\mu}\right)\right] \\
& \cdot\left(\sup _{t_{0}-\bar{\tau} \leq s \leq t_{0}+\bar{\tau}} \mathbb{E}|y(s)|^{p}\right) \\
& =: c_{4} \int_{t_{0}}^{t} \mathbb{E}|x(s)-y(s)|^{p} \mathrm{~d} s \\
& +c_{5}\left(\sup _{t_{0}+\bar{\tau} \leq s \leq t_{0}-\bar{\tau}+2 \Delta} \mathbb{E}|y(s)|^{p}\right) \\
& +c_{6}\left(\sup _{t_{0}-\bar{\tau} \leq s \leq t_{0}+\bar{\tau}} \mathbb{E}|y(s)|^{p}\right) \\
& \leq c_{4} \int_{t_{0}}^{t} \mathbb{E}|x(s)-y(s)|^{p} \mathrm{~d} s \\
& +c_{5}\left(\sup _{t_{0}-\bar{\tau}+\Delta \leq s \leq t_{0}-\bar{\tau}+2 \Delta} \mathbb{E}|y(s)|^{p}\right)+\left(c_{5}+c_{6}\right) \\
& \cdot\left(\sup _{t_{0}-\bar{\tau} \leq s \leq t_{0}-\bar{\tau}+\Delta} \mathbb{E}|y(s)|^{p}\right),
\end{aligned}
$$$$
c_{6}=4^{p-1} c_{1} c_{2}+\frac{c_{1} c_{3}}{8 p \beta}(8 \alpha)^{p}
$$$$
+\left(8^{p-1} c_{1} \bar{\tau}+4^{p-1} k^{p}\right)\left(1+\frac{1}{1-\mu}\right) .
$$

When $t_{0}+\bar{\tau} \leq t \leq t_{0}+2 \Delta$, by applying the Gronwall inequality, we get

$$
\begin{aligned}
& \mathbb{E}|x(t)-y(t)|^{p} \\
& \leq c_{5} \exp \left(2 \Delta c_{4}\right)\left(\sup _{t_{0}-\bar{\tau}+\Delta \leq s \leq t_{0}-\bar{\tau}+2 \Delta} \mathbb{E}|y(s)|^{p}\right) \\
& \quad+\left(c_{5}+c_{6}\right) \exp \left(2 \Delta c_{4}\right)\left(\sup _{t_{0}-\bar{\tau} \leq s \leq t_{0}-\bar{\tau}+\Delta} \mathbb{E}|y(s)|^{p}\right) .
\end{aligned}
$$


Therefore, when $t_{0}-\bar{\tau}+\Delta \leq s \leq t_{0}-\bar{\tau}+2 \Delta$, then

$$
\begin{aligned}
& \cdot\left(\sup _{t_{0}-\bar{\tau} \leq s \leq t_{0}-\bar{\tau}+\Delta} \mathbb{E}|y(s)|^{p}\right)+2^{p-1} c_{5} \exp \left(2 \Delta c_{4}\right) \\
& \cdot\left(\sup _{t_{0}-\bar{\tau}+\Delta \leq s \leq t_{0}-\bar{\tau}+2 \Delta} \mathbb{E}|y(s)|^{p}\right) .
\end{aligned}
$$$$
\mathbb{E}|y(t)|^{p} \leq 2^{p-1} \mathbb{E}|x(t)-y(t)|^{p}+2^{p-1} \mathbb{E}|x(t)|^{p}
$$$$
\leq\left[2^{p-1}\left(c_{5}+c_{6}\right) \exp \left(2 \Delta c_{4}\right)\right.
$$

$\left.+2^{p-1} \alpha^{p} \exp \left(-p \beta\left(t-t_{0}\right)\right)\right]$

From (12), if $\bar{\tau}<\widetilde{\tau}, 1-2^{p-1} c_{5} \exp \left(2 \Delta c_{4}\right)>0$, we have

$$
\sup _{t_{0}-\bar{\tau}+\Delta \leq s \leq t_{0}-\bar{\tau}+2 \Delta} \mathbb{E}|y(s)|^{p} \leq \frac{2^{p-1}\left(c_{5}+c_{6}\right) \exp \left(2 \Delta c_{4}\right)+2^{p-1} \alpha^{p} \exp \left(-p \beta\left(t-t_{0}\right)\right)}{1-2^{p-1} c_{5} \exp \left(2 \Delta c_{4}\right)}\left(\sup _{t_{0}-\bar{\tau} \leq s \leq t_{0}-\bar{\tau}+\Delta} \mathbb{E}|y(s)|^{p}\right),
$$

that is,

$$
\begin{aligned}
\mathbb{E}|y(t)|^{p} & \leq \frac{2^{p-1}\left(c_{5}+c_{6}\right) \exp \left(2 \Delta c_{4}\right)+2^{p-1} \alpha^{p} \exp (-p \beta(\Delta-\bar{\tau}))}{1-2^{p-1} c_{5} \exp \left(2 \Delta c_{4}\right)}\left(\sup _{t_{0}-\bar{\tau} \leq s \leq t_{0}-\bar{\tau}+\Delta} \mathbb{E}|y(s)|^{p}\right) \\
& =: F(k, \bar{\tau})\left(\sup _{t_{0}-\bar{\tau} \leq s \leq t_{0}-\bar{\tau}+\Delta} \mathbb{E}|y(s)|^{p}\right),
\end{aligned}
$$

where

$$
\text { If } \bar{\tau}=0 \text {, then }
$$

$$
\begin{aligned}
& F(k, \bar{\tau}) \\
& =\frac{2^{p-1}\left(c_{5}+c_{6}\right) \exp \left(2 \Delta c_{4}\right)+2^{p-1} \alpha^{p} \exp (-p \beta(\Delta-\bar{\tau}))}{1-2^{p-1} c_{5} \exp \left(2 \Delta c_{4}\right)} .
\end{aligned}
$$

$$
F(k, 0)=\frac{2^{p-1}\left[c_{5}+4^{p-1} k^{p}(1+1 /(1-\mu))\right] \exp \left(2 \Delta\left(4^{p-1}+2^{p-1}\right) c_{1}\right)+2^{p-1} \alpha^{p} \exp (-p \beta \Delta)}{1-2^{p-1} c_{5} \exp \left(2 \Delta\left(4^{p-1}+2^{p-1}\right) c_{1}\right)} .
$$

Obviously,

$$
1-2^{p-1} c_{5} \exp \left(2 \Delta\left(4^{p-1}+2^{p-1}\right) c_{1}\right)>0,
$$

as $0<k^{p}<k<\exp \left(-2 \Delta\left(4^{p-1}+2^{p-1}\right) c_{1}\right) /\left(2^{p-1} c_{1} \Delta 12^{p-1} 2^{p}(1 /\right.$ $\left.(1-\mu)+1 /(1-2 \mu))+8^{p-1} /(1-\mu)\right)$.

Moreover, $\partial F(k, 0) / \partial k>0$. Therefore, $F(k, 0)$ is strictly increasing with respect to $k$. Consequently, by the root existence theorem of continuous functions, $F(k, 0)=0$ has an unique positive root $2 \widetilde{k}$.

When $t_{0}-\bar{\tau}+\Delta \leq s \leq t_{0}-\bar{\tau}+2 \Delta$, let

$$
\frac{\partial \ln F(k, \bar{\tau})}{\partial \varepsilon}=0 \text {. }
$$

We have

$$
\begin{aligned}
& {\left[\frac{\partial}{\partial \varepsilon}\left(c_{5}+c_{6}\right)+2 \Delta\left(c_{5}+c_{6}\right) \frac{\partial}{\partial \varepsilon} c_{4}\right](1} \\
& \left.-2^{p-1} c_{5} \exp \left(2 \Delta c_{4}\right)\right)+\left[2^{p-1}\left(c_{5}+c_{6}\right) \exp \left(2 \Delta c_{4}\right)\right.
\end{aligned}
$$

$$
\begin{aligned}
& \left.+2^{p-1} \alpha^{p} \exp \left(-p \beta\left(t-t_{0}\right)\right)\right]\left[\frac{\partial}{\partial \varepsilon} c_{5}+2 \Delta c_{5} \frac{\partial}{\partial \varepsilon} c_{4}\right] \\
& =0
\end{aligned}
$$

that is,

$$
\begin{aligned}
& \left\{\left[\left(c_{51}+c_{61}\right)+2 \Delta c_{41} 4^{p-1} k^{p}\left(1+\frac{2}{1-\mu}\right)\right]\right. \\
& \quad+c_{1}\left[2 \Delta c_{41}\left(c_{51}+c_{61}\right)\right. \\
& \left.\quad+\Delta c_{41} c_{51}(2 \alpha)^{p} \exp (-p \beta(\Delta-\bar{\tau}))\right] \\
& \quad+\left[c_{51} 8^{p-1} k^{p}\left(1+\frac{2}{1-\mu}\right)-\left(c_{51}+c_{61}\right) \frac{8^{p-1} k^{p}}{1-\mu}\right] \\
& \quad \cdot \exp \left(2 \Delta c_{41} c_{1}\right)+\left[2^{p-1} c_{51}+2 \Delta c_{41} \frac{8^{p-1} k^{p}}{1-\mu}\right] \alpha^{p}
\end{aligned}
$$




$$
\begin{aligned}
& \left.\cdot \exp \left(-p \beta\left(t-t_{0}\right)\right)\right\}\left[(4 \Delta)^{p-1}\left\|\bar{A}_{i}\right\|^{p} K_{1}^{p}(1-p) \frac{1}{\varepsilon^{p}}\right. \\
& \left.+\frac{1}{4 \Delta}[4 \Delta p(p-1)]^{p / 2}\left\|\bar{B}_{i}\right\|^{p} K_{2}^{p}(1-p) \frac{-1}{(1-\varepsilon)^{p}}\right] \\
& =0
\end{aligned}
$$

where

$$
\begin{aligned}
& c_{12}=(4 \Delta)^{p-1}\left\|\bar{A}_{i}\right\|^{p} K_{1}^{p}, \\
& c_{13}=\frac{1}{4 \Delta}[4 \Delta p(p-1)]^{p / 2}\left\|\bar{B}_{i}\right\|^{p} K_{2}^{p}, \\
& c_{41}=4^{p-1}+2^{p-1}+8^{p-1} c_{3}, \\
& c_{51}=12^{p-1} \Delta(2 k)^{p}\left(\frac{1}{1-\mu}+\frac{1}{1-2 \mu}\right), \\
& c_{61}=4^{p-1} c_{2}+\frac{c_{3}}{8 p \beta}(8 \alpha)^{p}+8^{p-1} \bar{\tau}\left(1+\frac{1}{1-\mu}\right) .
\end{aligned}
$$

Thus, we further have

$$
\begin{aligned}
& {\left[(4 \Delta)^{p-1}\left\|\bar{A}_{i}\right\|^{p}\left(-K_{1}\right)^{p}\right.} \\
& \left.\quad-\frac{1}{4 \Delta}[4 \Delta p(p-1)]^{p / 2}\left\|\bar{B}_{i}\right\|^{p} K_{2}^{p}\right] \varepsilon^{p}+(4 \Delta)^{p-1}
\end{aligned}
$$

$$
\begin{aligned}
& \cdot\left\|\bar{A}_{i}\right\|^{p} K_{1}^{p}(-1)^{p-1} \varepsilon^{p-1}+\cdots+(4 \Delta)^{p-1}\left\|\bar{A}_{i}\right\|^{p} K_{1}^{p} \\
& =0 \\
\text { or } & {\left[\left(c_{51}+c_{61}\right)+2 \Delta c_{41} 4^{p-1} k^{p}\left(1+\frac{2}{1-\mu}\right)\right] } \\
& +\left[c_{12} \varepsilon^{1-p}+c_{13}(1-\varepsilon)^{1-p}\right]\left[2 \Delta c_{41}\left(c_{51}+c_{61}\right)\right. \\
& \left.+\Delta c_{41} c_{51}(2 \alpha)^{p} \exp \left(-p \beta\left(t-t_{0}\right)\right)\right] \\
& +\left[c_{51} 8^{p-1} k^{p}\left(1+\frac{2}{1-\mu}\right)-\left(c_{51}+c_{61}\right) \frac{8^{p-1} k^{p}}{1-\mu}\right] \\
& \cdot \exp \left(2 \Delta c_{41} c_{1}\right)+\left[2^{p-1} c_{51}+2 \Delta 2^{p-1} c_{41} \frac{4^{p-1} k^{p}}{1-\mu}\right] \alpha^{p} \\
& \cdot \exp (-p \beta(\Delta-\bar{\tau}))=0 .
\end{aligned}
$$

From (38), it is easy to see that there exists a unique $\bar{\tau}$ such that $\bar{\tau}=\tau_{\max }$, when $\varepsilon \in(0,1)$.

Choosing $\gamma=-\ln F(k, \bar{\tau}) / \Delta$, we have

$$
\begin{aligned}
& \sup _{t_{0}-\bar{\tau}+\Delta \leq t \leq t_{0}-\bar{\tau}+2 \Delta} \mathbb{E}\left|y\left(t ; t_{0} ; \psi\right)\right|^{p} \\
& \quad \leq \exp (-\gamma \Delta)\left(\sup _{t_{0}-\bar{\tau} \leq t \leq t_{0}-\bar{\tau}+\Delta} \mathbb{E}\left|y\left(t ; t_{0} ; \psi\right)\right|^{p}\right) .
\end{aligned}
$$

Then, for any positive integer $m=1,2, \ldots$, by the existence and uniqueness of the state of system (2), when $t \geq t_{0}-\bar{\tau}+(m-1) \Delta$, we have

$$
\begin{aligned}
& y\left(t ; t_{0}, \psi\right)=y\left(t ; t_{0}-\bar{\tau}\right. \\
& \left.\quad+(m-1) \Delta, \tilde{y}\left(t_{0}-\bar{\tau}+(m-1) \Delta ; t_{0}, \psi\right)\right) .
\end{aligned}
$$

From (39) and (40), we have

$$
\begin{aligned}
& \sup _{t_{0}-\bar{\tau}+m \Delta \leq t \leq t_{0}-\bar{\tau}+(m+1) \Delta} \mathbb{E}\left|y\left(t ; t_{0} ; \psi\right)\right|^{p} \\
& \quad=\left(\sup _{t_{0}-\bar{\tau}+(m-1) \Delta+\Delta \leq t \leq t_{0}-\bar{\tau}+(m-1) \Delta+2 \Delta} \mathbb{E}\left|y\left(t ; t_{0}-\bar{\tau}+(m-1) \Delta, \tilde{y}\left(t_{0}-\bar{\tau}+(m-1) \Delta ; t_{0}, \psi\right)\right)\right|^{p}\right) \\
& \quad \leq \exp (-\gamma \Delta)\left(\sup _{t_{0}-\bar{\tau}+(m-1) \Delta \leq t \leq t_{0}-\bar{\tau}+m \Delta} \mathbb{E}\left|y\left(t ; t_{0} ; \psi\right)\right|^{p}\right) \leq \cdots \leq \exp (-\gamma m \Delta)\left(\sup _{t_{0}-\bar{\tau} \leq t \leq t_{0}-\bar{\tau}+\Delta} \mathbb{E}\left|y\left(t ; t_{0} ; \psi\right)\right|^{p}\right) \\
& \quad=c \exp (-\gamma m \Delta),
\end{aligned}
$$

where $c=\sup _{t_{0}-\bar{\tau} \leq t \leq t_{0}-\bar{\tau}+\Delta} \mathbb{E}\left|y\left(t ; t_{0} ; \psi\right)\right|^{p}$. Thus, for $\forall t \geq t_{0}+\Delta$, and for the fact that there exists a positive integer $m$ such that $t_{0}-\bar{\tau}+m \Delta \leq t \leq t_{0}-\bar{\tau}+(m+1) \Delta$, we have

$$
\begin{aligned}
\mathbb{E}\left|y\left(t ; t_{0} ; \psi\right)\right|^{p} & \leq c \exp \left(-\gamma t+\gamma t_{0}+\gamma \Delta\right) \\
& =c \exp (\gamma \Delta) \exp \left(-\gamma\left(t-t_{0}\right)\right),
\end{aligned}
$$

and (42) is also true when $t_{0}-\bar{\tau} \leq t \leq t_{0}-\bar{\tau}+\Delta$. Therefore, system (2) is $p$ th moment global exponential stable. According to Lemma 6, system (2) is also almost surely global exponential stable.

Corollary 9. When $p=2$ and $\varepsilon=1 / 2$, system (2) is mean square global exponential stable and also almost surely global 
exponential stable, if $k<\min \left\{\widetilde{k}, \exp \left(-12 \Delta \widetilde{c}_{1}\right) /\left(96 \Delta \widetilde{c}_{1}(1 /(1-\right.\right.$ $\mu)+1 /(1-2 \mu))+8 /(1-\mu))\}, \bar{\tau}<\widetilde{\tau}$, where $2 \widetilde{k}$ is a unique positive solution of the transcendental equation:

$$
\begin{aligned}
& {\left[192 \Delta k^{2}\left(\frac{1}{1-\mu}+\frac{1}{1-2 \mu}\right) \widetilde{c}_{1}+8 k^{2}\left(1+\frac{3}{1-\mu}\right)\right]} \\
& \cdot \exp \left(12 \Delta \widetilde{c}_{1}\right)+2 \alpha^{2} \exp (-2 \beta \Delta)=1,
\end{aligned}
$$

and $\tilde{\tau}$ is a unique positive solution of the transcendental equation:

$$
\begin{aligned}
& {\left[\left(192 \Delta k^{2}\left(\frac{1}{1-\mu}+\frac{1}{1-2 \mu}\right)+16 \bar{\tau}\left(1+\frac{1}{1-\mu}\right)\right.\right.} \\
& \left.\left.\quad+8 \widetilde{c}_{2}+\frac{8 \widetilde{c}_{3} \alpha^{2}}{\beta}\right) \widetilde{c}_{1}+8 k^{2}\left(1+\frac{3}{1-\mu}\right)\right] \\
& \quad \cdot \exp \left(4 \Delta\left(3+4 \widetilde{c}_{3}\right) \widetilde{c}_{1}\right)+2 \alpha^{2} \exp (-2 \beta(\Delta-\bar{\tau})) \\
& \quad=1
\end{aligned}
$$

where

$$
\begin{aligned}
\widetilde{c}_{1}= & 8 \Delta\left\|\bar{A}_{i}\right\|^{2} K_{1}^{2}+4\left\|\bar{B}_{i}\right\|^{2} K_{2}^{2}, \\
\widetilde{c}_{2}= & 6 k^{2} \bar{\tau}\left(\frac{1}{1-\mu}+\frac{2}{1-2 \mu}\right) \\
& +\left(6 \bar{\tau}\left\|\bar{A}_{i}\right\|^{2} K_{1}^{2}+6\left\|\bar{B}_{i}\right\|^{2} K_{2}^{2}\right) \frac{\bar{\tau}^{2}}{1-\mu}, \\
\widetilde{c}_{3}= & 6 \bar{\tau}\left(\bar{\tau}\left\|\bar{A}_{i}\right\|^{2} K_{1}^{2}+\left\|\bar{B}_{i}\right\|^{2} K_{2}^{2}\right)\left(1+\frac{1}{1-\mu}\right) .
\end{aligned}
$$

$\Delta$ is a step; $\Delta-\bar{\tau}>\ln \left(2 \alpha^{2}\right) /(2 \beta)>0$.

Remark 10. Theorem 8 shows that when system (6) without neutral terms and time-varying delays is globally exponentially stable, system (2) induced by neutral terms and timevarying delays can be $p$ th moment globally exponentially stable and almost surely globally exponentially stable if the neutral terms are sufficiently small and the time-varying delays are smaller than the given upper bounds.

Remark 11. It shows that our results generalize and improve the corresponding results of recent works in Theorem 8. From the proofs of Theorem 8 , we can see that the upper bounds of contraction coefficients of neutral terms and time-varying delays can be estimated by solving transcendental equation. The two transcendental equations in Theorem 8 can be solved by using some software such as MATLAB; the condition in Theorem 8 can be verified easily.

\section{Numerical Example}

In this section, we give an example with numerical simulation to illustrate our result in the preceding section.
Example 1. Consider a two-state neutral type delay system:

$$
\begin{aligned}
\mathrm{d}\left(\begin{array}{l}
x_{1} \\
x_{2}
\end{array}\right)= & K_{1} A(r(t))\left(\begin{array}{l}
x_{1} \\
x_{2}
\end{array}\right) \mathrm{d} t \\
& +K_{2} B(r(t))\left(\begin{array}{l}
\sin \left(x_{2}\right) \\
\sin \left(x_{1}\right)
\end{array}\right) \mathrm{d} w(t),
\end{aligned}
$$

where $K_{1}=0.03, K_{2}=0.01$, and the generators of the Markov chain and parameters are

$$
\begin{aligned}
\Gamma & =\left(\begin{array}{cc}
-2 & 2 \\
1 & -1
\end{array}\right), \\
A(1) & =\left(\begin{array}{ll}
2 & 0 \\
0 & 1
\end{array}\right), \\
A(2) & =\left(\begin{array}{ll}
1 & 0 \\
0 & 0.5
\end{array}\right), \\
B(1) & =\left(\begin{array}{cc}
0 & 0.05 \\
0.1 & 0
\end{array}\right), \\
B(2) & =\left(\begin{array}{cc}
0 & 0.5 \\
0.5 & 0
\end{array}\right) .
\end{aligned}
$$

According to [20], system (46) is globally exponentially stable with $\alpha=0.8$ and $\beta=0.5$.

In the presence of neutral terms and time-varying delays, the system with Markovian switching becomes

$$
\begin{aligned}
& \mathrm{d}\left(\begin{array}{l}
y_{1}-k \cos \left(y_{1}(t-\tau(t))\right) \\
y_{2}-k \cos \left(y_{2}(t-\tau(t))\right)
\end{array}\right) \\
& =K_{1} A(r(t))\left(\begin{array}{l}
y_{1}(t-\tau(t)) \\
y_{2}(t-\tau(t))
\end{array}\right) \mathrm{d} t \\
& \quad+K_{2} B(r(t))\left(\begin{array}{c}
\sin \left(y_{2}(t-\tau(t))\right) \\
\sin \left(y_{1}(t-\tau(t))\right)
\end{array}\right) \mathrm{d} w(t),
\end{aligned}
$$

where $\tau(t)$ is the time-varying delay and $k \in(0,1)$.

Figure 1 depicts the Markov chain with the generator $\Gamma$.

Let $\Delta=0.5, \mu=0.9$, by Corollary 9; choosing $\varepsilon=1 / 2$, then (43) and (44) become

$$
\begin{aligned}
& \left(12.18 k^{2}+248 k^{2}\right) \exp (6 \times 0.0145)+1.28 \exp (-0.5 \\
& +\bar{\tau})=1 \\
& {[0.0145(0.336+176 \bar{\tau}} \\
& \quad+8\left(0.018 \bar{\tau}+0.216 \bar{\tau}^{3}+0.0015 \bar{\tau}^{2}\right) \\
& +10.24 \bar{\tau}(0.2376 \bar{\tau}+0.0017))+0.0992] \\
& \quad \cdot \exp (0.029(3+4 \bar{\tau}(0.2376 \bar{\tau}+0.0017)))+1.28 \\
& \quad \cdot \exp (-0.5+\bar{\tau})=1
\end{aligned}
$$




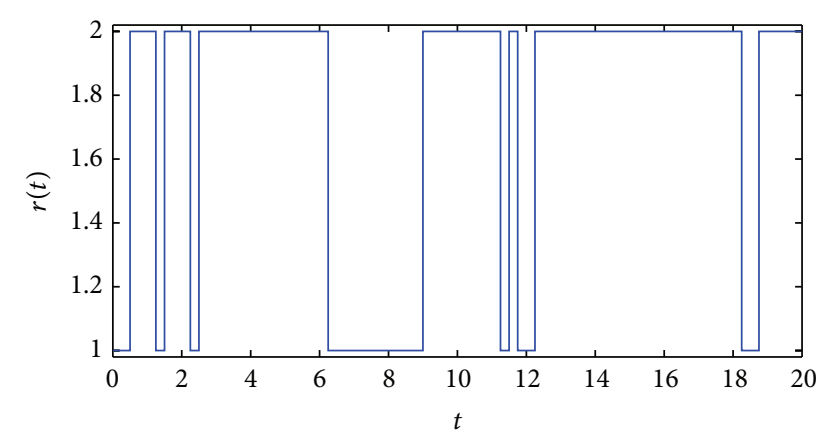

Figure 1: Simulation of Markov chain with the generator $\Gamma$ in example.

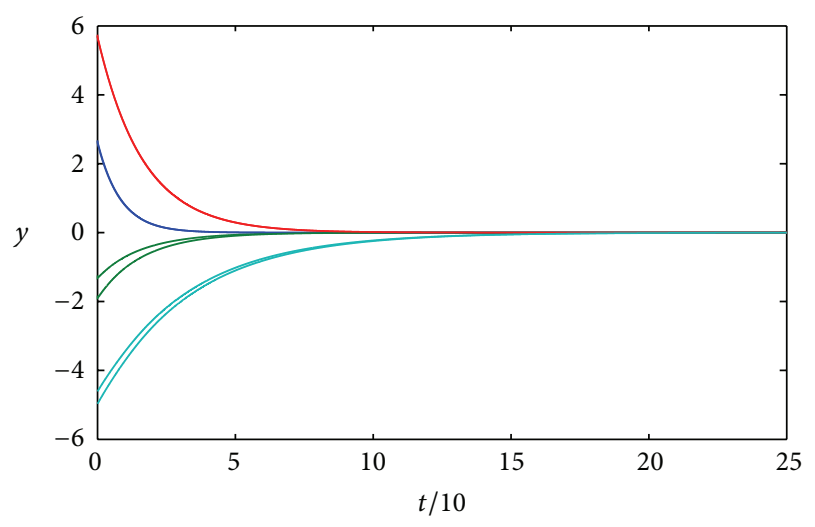

FIGURE 2: Stable states of the system in (48) with $\bar{\tau}=0.03$ and $k=$ 0.02 .

which have the solution $\widetilde{k} \approx 0.0281, \widetilde{\tau} \approx 0.0366$, and $\Delta-\widetilde{\tau}=$ $0.4634>\ln (1.28)=0.2469$. Thus, system (48) is globally exponentially stable (see, Figure 2 , when $\bar{\tau}=0.03, k=0.02$ ).

\section{Conclusion}

In this paper, the robust stability of hybrid stochastic neural networks subject to neutral terms and time-varying delays is analyzed. In order to maintain global exponential stability of neural networks, the upper bounds of contraction coefficients of neutral terms and time-varying delays are derived. The results herein are feasible and provide the design and application of neural network with a theoretical basis. Some future directions may be aimed at the improvements of the upper bounds of contraction coefficients of neutral terms and timevarying delays. We will continue to study the robust stability using the Lyapunov theory or linear matrix inequality to improve stability condition.

\section{Conflict of Interests}

The authors declare that there is no conflict of interests regarding the publication of this paper.

\section{Acknowledgments}

The authors would like to thank the referee and the associate editor for their very useful comments and suggestions. The authors would also like to thank the financial supports from the National Natural Science Foundation of China (nos. 61374085 and 61304068), Huazhong Agricultural University Scientific \& Technological Self-Innovation Foundation under Grant no. 2662014BQ059, Science and Technology Research Projects from the Education Department of Guangxi Zhuang Autonomous Region (no. KY2015LX592), China.

\section{References}

[1] F. Jiang, H. Yang, and Y. Shen, "On the robustness of global exponential stability for hybrid neural networks with noise and delay perturbations," Neural Computing \& Applications, vol. 24, no. 7-8, pp. 1497-1504, 2014.

[2] W. W. Luo, K. Zhong, S. Zhu et al., "Further results on robustness analysis of global exponential stability of recurrent neural networks with time delays and random disturbances," Neural Networks, vol. 53, pp. 127-133, 2014.

[3] S. Weiwei and C. Yiming, "New results on the robust stability analysis of neural networks with discrete and distributed time delays," Journal of Systems Engineering and Electronics, vol. 19, no. 3, pp. 592-597, 2008.

[4] Y. Shen and J. Wang, "Robustness analysis of global exponential stability of recurrent neural networks in the presence of time delays and random disturbances," IEEE Transactions on Neural Networks and Learning Systems, vol. 23, no. 1, pp. 87-96, 2012.

[5] Y. Shen and J. Wang, "Robustness analysis of global exponential stability of non-linear systems with time delays and neutral terms," IET Control Theory \& Applications, vol. 7, no. 9, pp. 12271232,2013

[6] D. Liberzon, Switching in Systems and Control, Birkhäauser, Boston, Mass, USA, 2003.

[7] M. Hua, F. Deng, and Y. Peng, "Stability of stochastic neural networks with Markovian jumping parameters?" Journal of Systems Engineering and Electronics, vol. 20, no. 3, pp. 613-618, 2009.

[8] Z.-G. Wu, P. Shi, H. Su, and J. Chu, "Stability analysis for discrete-time Markovian jump neural networks with mixed time-delays," Expert Systems with Applications, vol. 39, no. 6, pp. 6174-6181, 2012.

[9] K. Yuan, J. Cao, and H.-X. Li, "Robust stability of switched Cohen-Grossberg neural networks with mixed time-varying delays," IEEE Transactions on Systems, Man, and Cybernetics, Part B: Cybernetics, vol. 36, no. 6, pp. 1356-1363, 2006.

[10] Y. Shen and J. Wang, "Almost sure exponential stability of recurrent neural networks with Markovian switching," IEEE Transactions on Neural Networks, vol. 20, no. 5, pp. 840-855, 2009.

[11] X. Y. Meng, F. Q. Deng, and H. Li, "Stability of stochastic switched epidemic systems with discrete or distributed time delay," Journal of Systems Engineering and Electronics, vol. 25, no. 4, pp. 660-670, 2014.

[12] Z. Wang, Y. Liu, and X. Liu, "State estimation for jumping recurrent neural networks with discrete and distributed delays," Neural Networks, vol. 22, no. 1, pp. 41-48, 2009. 
[13] S. Zhu, Y. Shen, and L. Liu, "Exponential stability of uncertain stochastic neural networks with markovian switching," Neural Processing Letters, vol. 32, no. 3, pp. 293-309, 2010.

[14] X. G. Wang, D. M. Zhang, and J. Liu, "Stability analysis of cellular neural networks with time-varying delay," Journal of Systems Engineering and Electronics, vol. 20, no. 2, pp. 266-273, 2009.

[15] S. Zhu, W. Luo, and Y. Shen, "Robustness analysis for connection weight matrices of global exponential stability of stochastic delayed recurrent neural networks," Circuits, Systems, and Signal Processing, vol. 33, no. 7, pp. 2065-2083, 2014.

[16] S. Zhu and Y. Shen, "Robustness analysis of global exponential stability of neural networks with Markovian switching in the presence of time-varying delays or noises," Neural Computing and Applications, vol. 23, no. 6, pp. 1563-1571, 2013.

[17] H. Huang, Y. Qu, and H.-X. Li, "Robust stability analysis of switched Hopfield neural networks with time-varying delay under uncertainty," Physics Letters, Section A: General, Atomic and Solid State Physics, vol. 345, no. 4-6, pp. 345-354, 2005.

[18] J. Xie, Y. Kao, C. Wang, and C. Gao, "Delay-dependent robust stability of uncertain neutral-type Itô stochastic systems with Markovian jumping parameters," Applied Mathematics and Computation, vol. 251, pp. 576-585, 2015.

[19] J. Xia, J. H. Park, and H. Zeng, "Improved delay-dependent robust stability analysis for neutral-type uncertain neural networks with Markovian jumping parameters and time-varying delays," Neurocomputing, vol. 149, pp. 1198-1205, 2015.

[20] X. Mao and C. Yuan, Stochastic Differential Equations with Markovian Switching, Imperial College Press, 2006. 


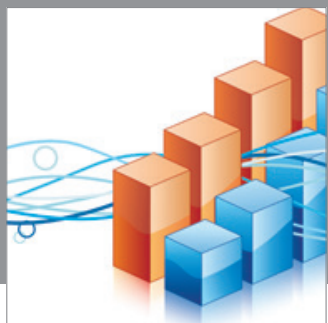

Advances in

Operations Research

mansans

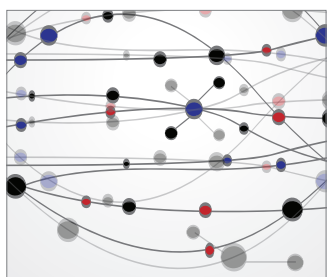

The Scientific World Journal
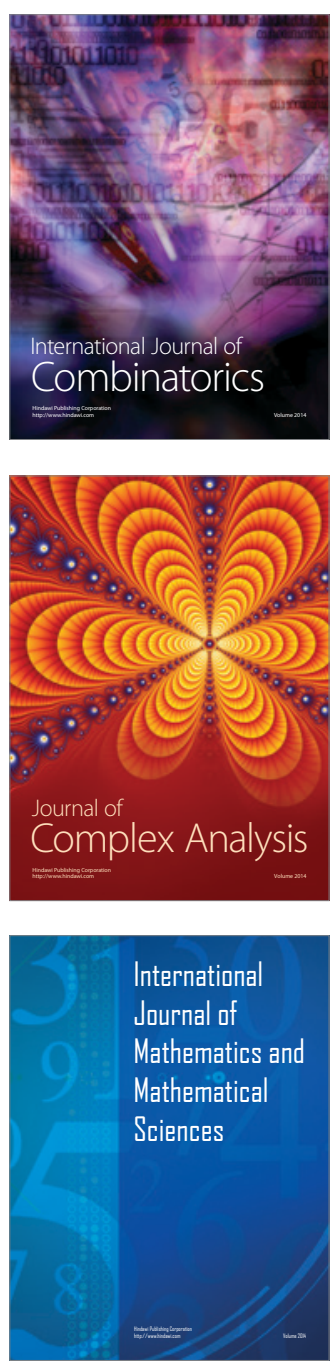
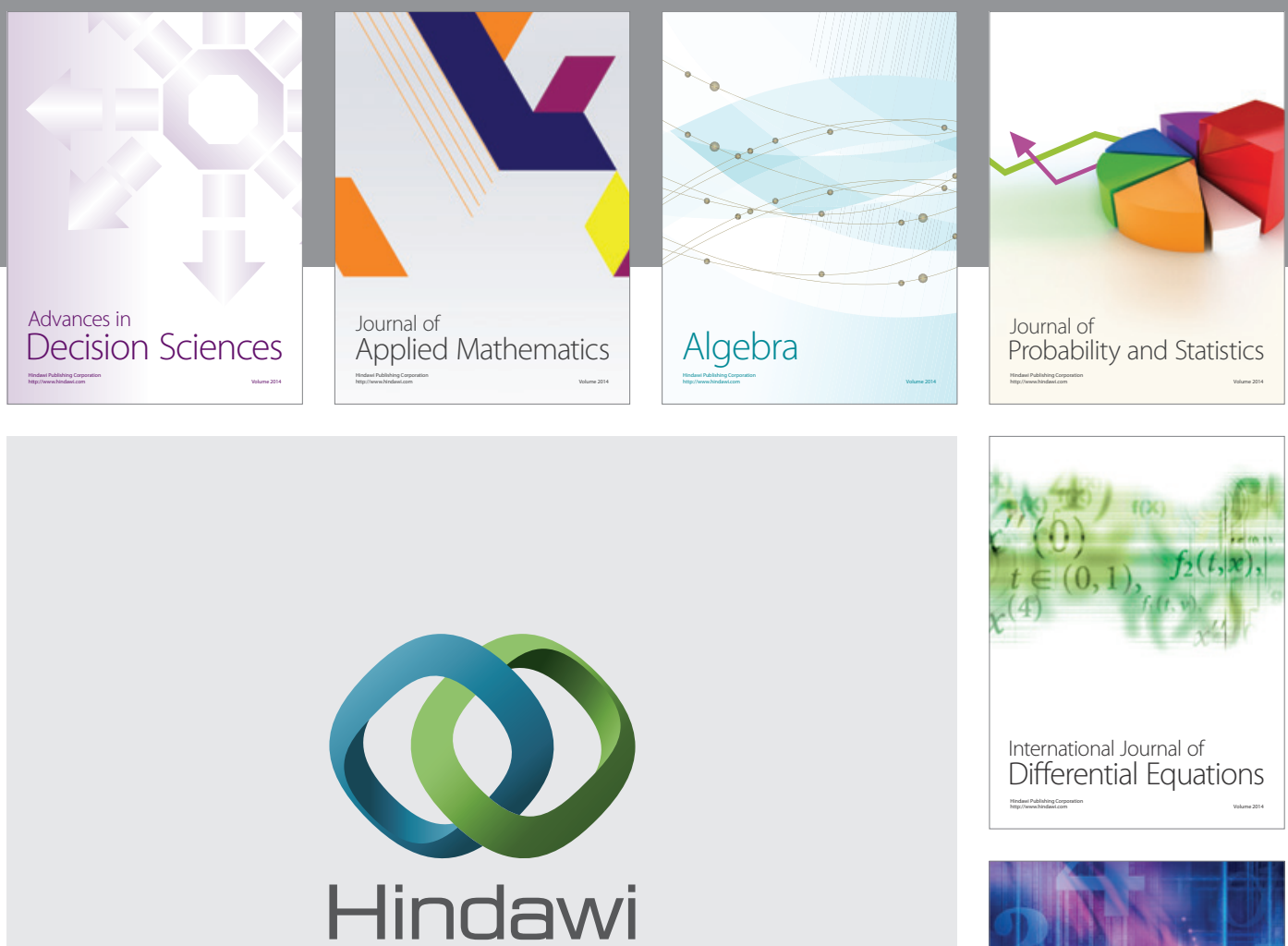

Submit your manuscripts at http://www.hindawi.com
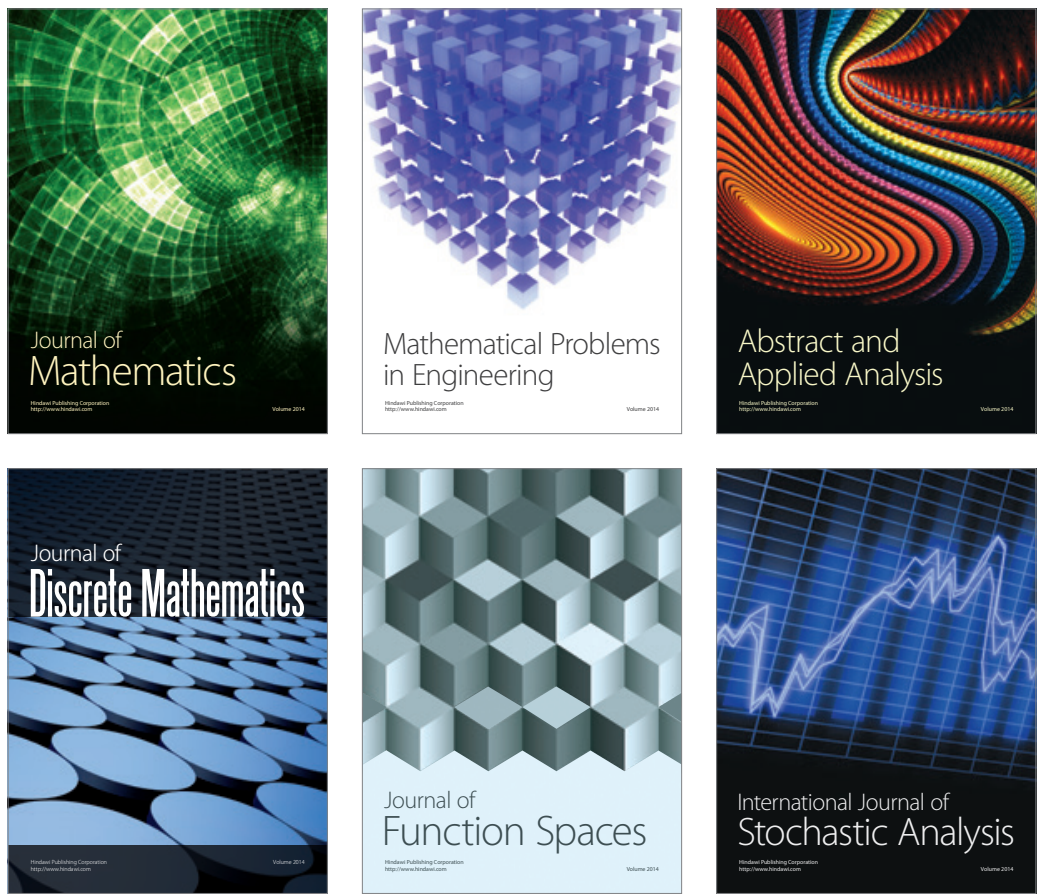

Journal of

Function Spaces

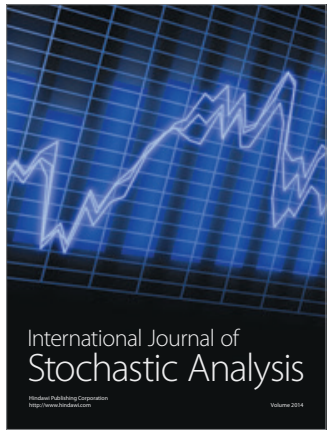

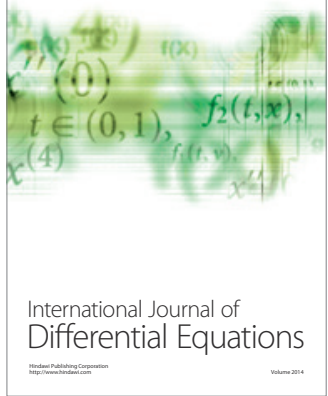
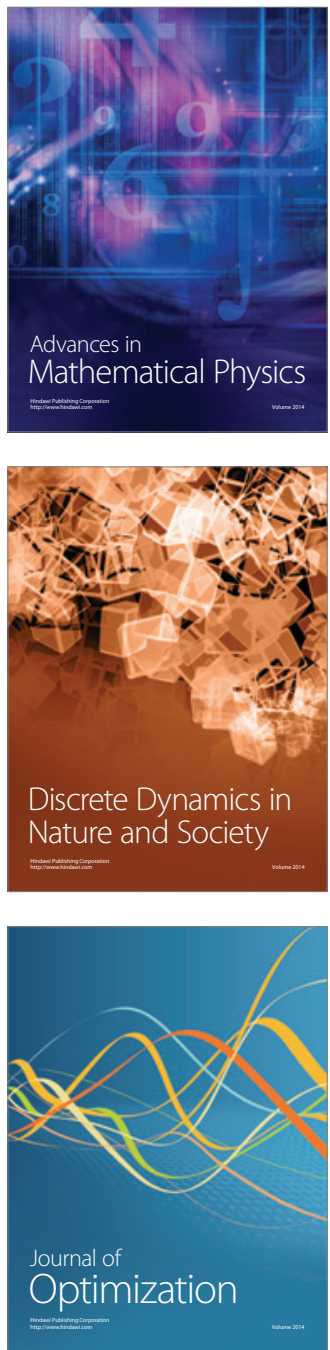